\title{
Material Identification Using a Bi-axial Test Machine
}

\author{
Paulo Flores ${ }^{1}$, Pierre Moureaux ${ }^{1}$ and Anne-Marie Habraken ${ }^{1}$ \\ ${ }^{1}$ M\&S Department, University of Liege, Chemin des Chevreuils 1, 4000 Liege, Belgium. \\ Paulo.Flores@ulg.ac.be
}

Keywords: Bi-axial machine, yield function and identification of material parameters.

\begin{abstract}
This paper shows the identification by mechanical tests of yield functions material parameters for a DC06 IF steel sheet of $0.8 \mathrm{~mm}$. The experimental equipments used are a tensile machine, a bi-axial machine able to perform plane-strain and simple shear tests separately or simultaneously and an optical strain gage. Tensile, plane-strain and simple shear tests were performed at $0^{\circ}, 45^{\circ}$ and $90^{\circ}$ from the sheet rolling direction in order to identify Hill 1948 and Hosford 1979 yield criteria. Two identification methods are used: one based on strain measurements and the other one based on stress measurements. The results show that mechanical tests reproducing other stress-states than tensile are required to obtain more accurate material parameters identification.
\end{abstract}

\section{Introduction}

The industrial requirements of quality, lightness, accuracy, resistance and economy have motivated a deeper study of metal and their forming processes. A good description of material behavior enables the prediction of several problems observed during metal forming processes such as wrinkles, earing and springback.

The constitutive laws that describe the material behavior can be obtained from crystallographic structure of the material or represented by phenomenological functions. Both approaches have advantages and drawbacks. This work is focused on the last approach due to the fact that they are implemented in most of the commercial finite elements codes and they can be identified by mechanical tests.

The number of the required mechanical tests is proportional to the complexity of the constitutive law. This article aims to identify two yield criteria for cold sheet metal forming using tensile, planestrain and simple shear tests.

The paper is divided in five sections. The first one describes the experimental equipments followed by the experimental results over the steel sheet. Third one describes the yield functions, and material parameters identification is shown in forth section. Finally some conclusions are established.

\section{Experimental set-up}

Bi-axial machine. A bi-axial machine able to perform plane-strain and simple shear tests, simultaneously or separately, was designed, built and validated at the M\&S Laboratory of the University of Liege (Fig. 1a). The arrows in Fig. 1a show the machine movement axes. The motion is generated by two hydraulic pistons, which can be controlled in force or displacement simultaneously or independently. The force capacity is $50 \mathrm{kN}$. The development and validation of this equipment can be seen in [1]. The original idea is based on the work done in [2].

Specimen. The specimen is shown in Figure $1 \mathrm{~b}$. The geometry is chosen in a way that the edge effect influence over the imposed force can be neglected, the strain field were homogeneous and the sliding between the grip wedge and the specimen were minimized $[1,3]$. 
Optical measurement system. The optical measurement system Aramis ${ }^{\circledR}$ is chosen to measure the deformations of the specimen. This system compares images taken in the deformed state with one taken as a reference in the undefomed state. The whole strain field is computed, enabling to identify the size of the homogeneous deformation zone. This system is also optimal for measuring complex strain-paths.

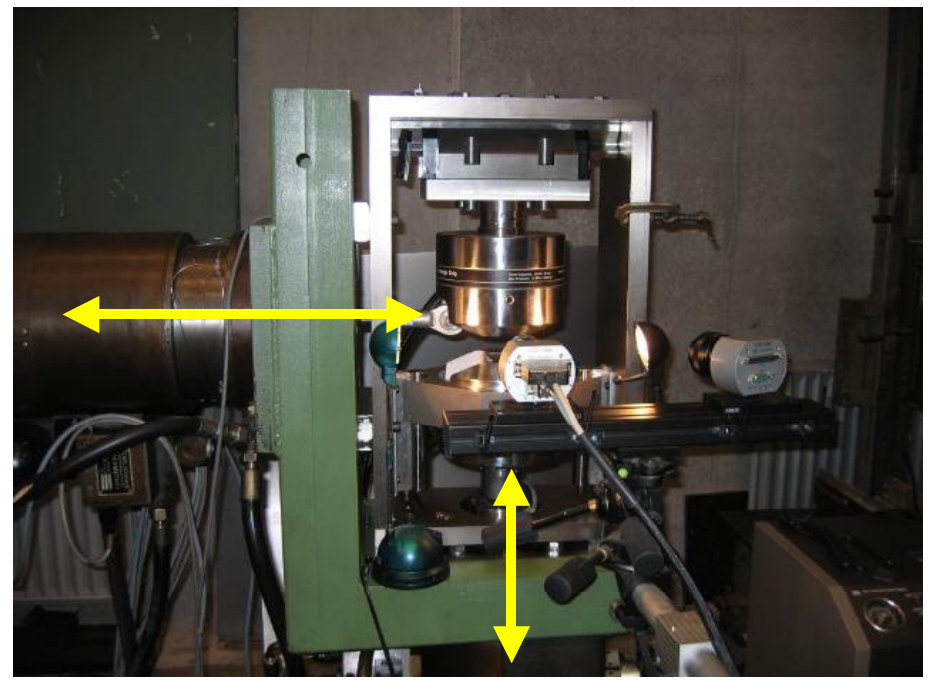

(a)

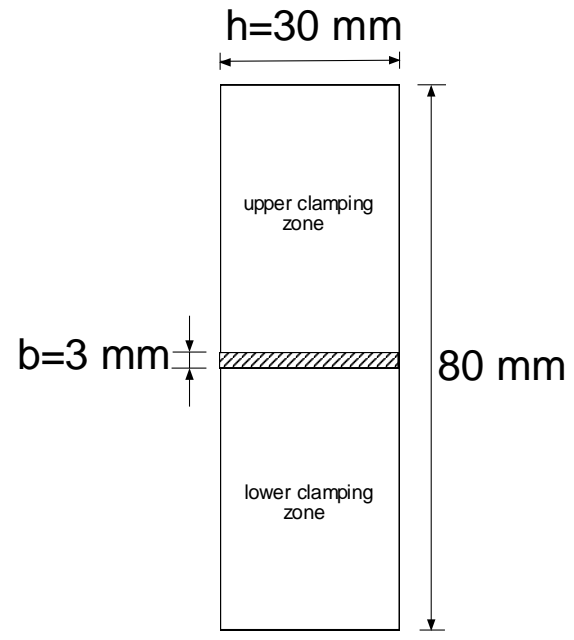

(b)

Figure 1: Experimental equipment. (a) Bi-axial machine with optical system. (b) Specimen geometry.

\section{Experimental results}

Three kinds of tests were performed: tensile, plane-strain and simple shear. Tensile tests were performed in a standard tensile test machine of $20 \mathrm{kN}$ capacity (with a normalized specimen) while the plane-strain and simple shear ones were performed in the bi-axial machine. For every case, specimens were obtained from a DC06 steel sheet of $0.8 \mathrm{~mm}$ of thickness at the rolling direction (RD), transversal to the rolling direction (TD) and at $45^{\circ}$ from the RD (see Fig. 2).

The tensile and the simple shear tests were performed at a constant plastic strain rate of $\mathscr{x}_{x}^{\prime}=1.8 \times 10^{-4} 1 / \mathrm{s}$ and $\not \not{X}^{\prime}=0.6 \times 10^{-3} 1 / \mathrm{s}$ respectively, while the plane-strain test at a constant speed of $0.005 \mathrm{~mm} / \mathrm{s}$.

The tests results are shown in Fig. 3. By symmetry, the simple shear test at TD is equal to the one at RD.

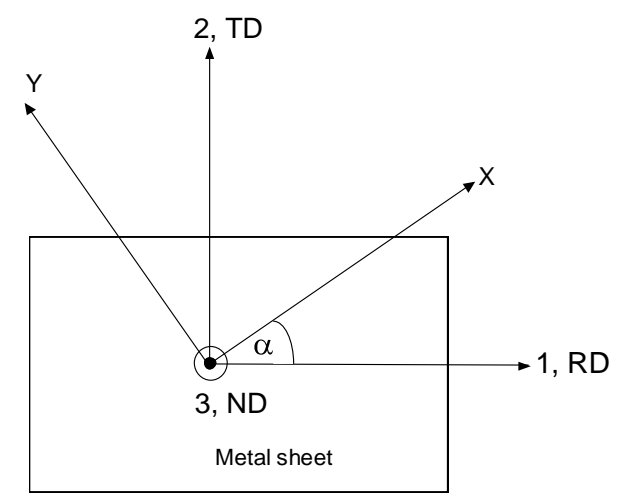

Figure 2: Axis definition for rolled metal sheet. 


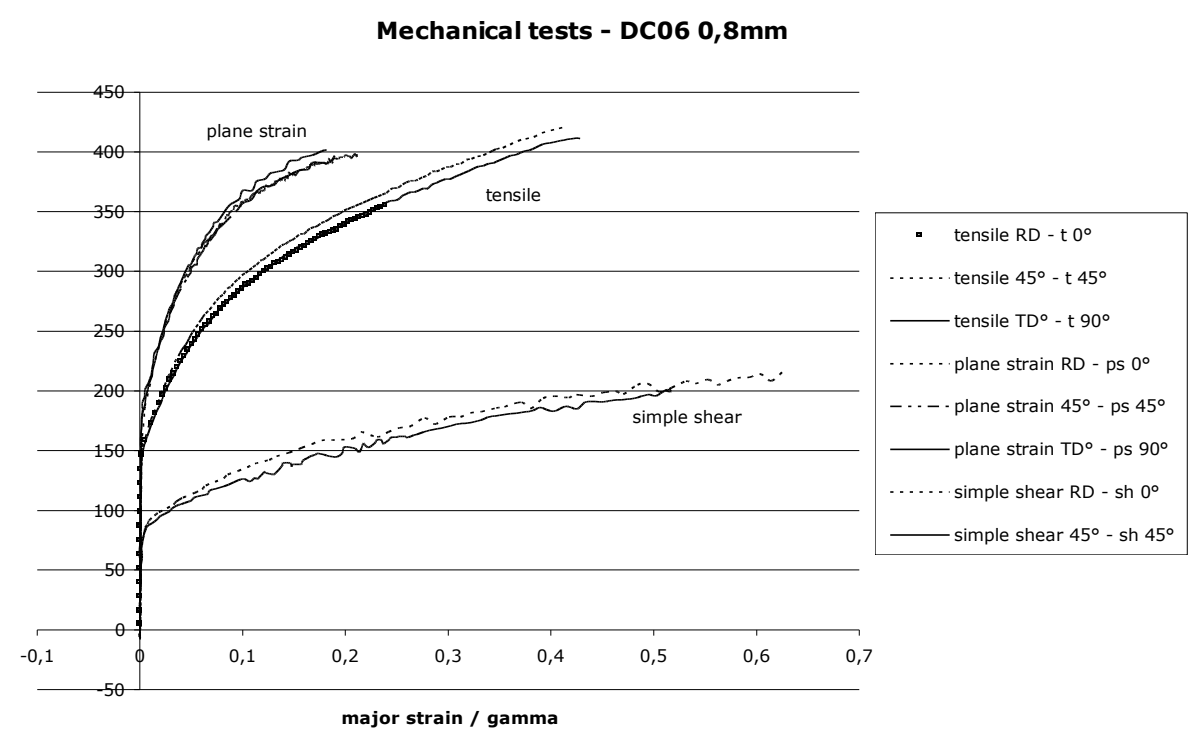

Figure 3: Tensile, plane-strain and simple shear tests for DC06 steel.

\section{Yield surfaces}

The two phenomenological yield criteria described in this section are commonly implemented in finite element codes and are usually identified by tensile tests. In both cases the material is supposed to have an anisotropy with three orthogonal symmetry planes.

Hill 1948 yield criterion. This quadratic yield criterion is a generalization of von Mises criterion.

$$
\Phi_{H i l l}(\underline{\sigma})=\frac{1}{2}\left[H\left(\sigma_{x x}-\sigma_{y y}\right)^{2}+G\left(\sigma_{x x}-\sigma_{z z}\right)^{2}+F\left(\sigma_{y y}-\sigma_{z z}\right)^{2}+2\left(N \sigma_{x y}^{2}+L \sigma_{x z}^{2}+M \sigma_{y z}^{2}\right)\right] .
$$

Hosford 1979 yield criterion. The main advantage of this non-quadratic yield criterion is that the fitting the value of the exponent ensures a good approximation of the experimental data [4]. Recommended values are $a=6$ for bcc materials and $a=8$ for fcc materials [5]. The main drawback is the lack of shear stress.

$$
\Phi_{\text {Hosford }}(\underline{\sigma})=H\left|\sigma_{x x}-\sigma_{y y}\right|^{a}+G\left|\sigma_{z z}-\sigma_{x x}\right|^{a}+F\left|\sigma_{y y}-\sigma_{z z}\right|^{a}
$$

Flow rule. The flow rule for associated plasticity (which is rather well confirmed by experiments for most ductile materials) becomes:

$$
\underline{\partial}=\not \dot{\gamma} \frac{\partial \Phi}{\partial \underline{\sigma}} .
$$

\section{Identification of material parameters}

For plane stresses the Eq. 2 and Eq. 3 can be simplified by assuming $\sigma_{z z}=\sigma_{x z}=\sigma_{y z}=0 ; \sigma_{x x} \neq 0$, $\sigma_{y y} \neq 0$ and $\sigma_{x y} \neq 0$. The initial equivalent flow stress is chosen to be the initial yield stress for a tensile test at the RD, i.e.:

$$
\Phi(\underline{\sigma})=\sigma_{0}^{2} .
$$


Lankford coefficients. These anisotropy coefficients are determined from three tensile tests, at RD, $\mathrm{TD}$ and $45^{\circ}$ from $\mathrm{RD}$. They represent the ratio between the transversal plastic strain rate and the thickness plastic strain rate. This last one is computed from conservation of volume, i.e.,

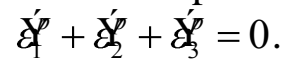

Using Eq. 3 the following equations can be established:

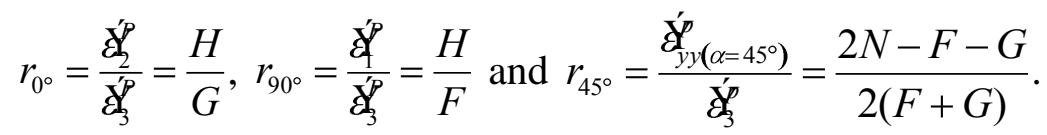

To complete the equations system, the relations $H+G=2$ for Hill 1948 and $H+G=1$ for Hosford 1979 are obtained respectively from Eq. 1 and Eq 2, together with Eq. 4.

The Lankford coefficient and the initial yield stress appear in Table 1 and the material parameters for both criteria appear in Tables 2 .

\begin{tabular}{|c|c|c|c|}
\hline$r_{0^{\circ}}$ & $r_{90^{\circ}}$ & $r_{45^{\circ}}$ & $\sigma_{0}\left(\varepsilon_{0}{ }^{\mathrm{P}}=0.2 \%\right)[\mathrm{MPa}]$ \\
\hline 1.98 & 2.56 & 1.67 & 142 \\
\hline
\end{tabular}

Table 1: Lankford coefficients and initial yield stress for DC06 $0.8 \mathrm{~mm}$.

\begin{tabular}{|l|c|c|c|c|c|}
\hline Material parameters & $H$ & $F$ & $G$ & $N$ & $a$ \\
\hline Hill 1948 & 1.33 & 0.52 & 0.67 & 2.58 & - \\
\hline Hosford 1979 & 0.66 & 0.26 & 0.34 & - & 6 \\
\hline
\end{tabular}

Table 2: Material parameters for DC06 steel $0.8 \mathrm{~mm}$ using Lankford coefficients.

Yield stresses at different plastic work. The plastic work $W_{p}$ at different plastic strain levels is computed from the tensile test at RD. For equal $W_{p}$ a set of $\left(\sigma_{\mathrm{xx}}, \sigma_{\mathrm{yy}}\right)$ is obtained from the other tests. Fig. 4 shows these points for the principal stresses plane. For small strain levels this points represent the yield surface [6].

Plastic contours DC06 0,8mm

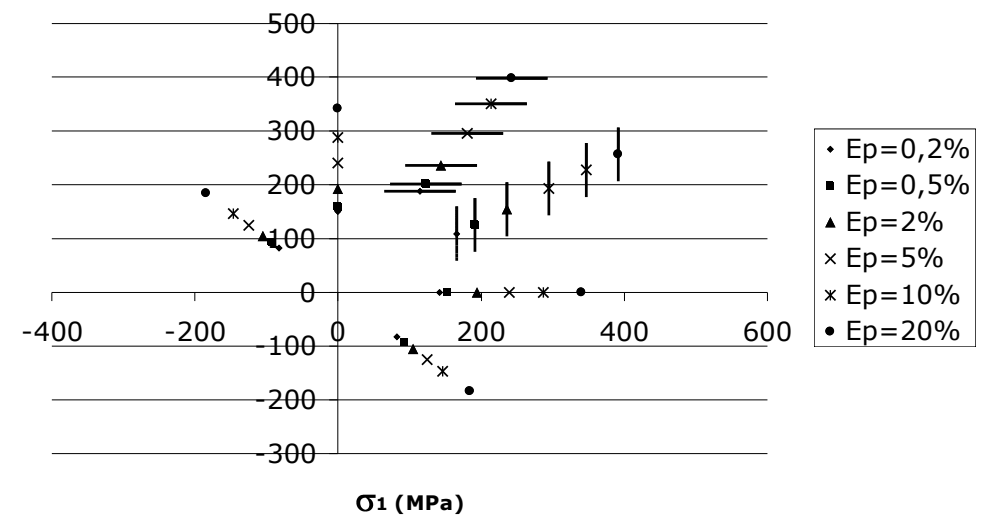

Figure 4: Contours of plastic work in the principal stress. Tensile and plane-strain tests at RD and $\mathrm{TD}$, simple shear tests at $45^{\circ}$ from RD.

The components of the plane-strain state are $\sigma_{1} \neq \sigma_{2} \neq 0$ and none of them negligible. The stress resulting from the impose load is the only one that can be measured, i.e., $\sigma_{1}$ for plane-strain test at $\mathrm{RD}$ and $\sigma_{2}$ for plane-strain at TD.

To establish a relation ship between the two components of the stress state, the Eq. 3, together with the fact that $\dot{y}_{2}^{\prime}=0$ for the test at RD and $\dot{x}^{\prime}=0$ for the one at TD, is used. 
The simple shear tests at $45^{\circ}$ from the RD represents a pure shear state at small strains. For moderately large strains the point remains a good approximation.

To fit the material parameters the following error function is minimized:

$$
\chi=\sum_{i=1, j}(1-\eta)\left[\frac{\sigma_{\Phi i}-\sigma_{\exp i}}{\sigma_{0 \exp }}\right]^{2}+\eta\left[r_{\Phi i}-r_{\exp i}\right]^{2}
$$

where sub-index $\Phi$ means the value deduced from the respective yield criterion, exp means values deduced from experiments and $j$ the number of tests. $\eta$ is a weighting factor defining the weight of stress and strain measurements. In what follows $\eta$ is set to 0 in order to focus the study in stress measurement.

Fig. 5 shows the Hill 1948 yield function fitted using 8 experimental points, while Fig. 6 compares the yield function shape in the principal stress directions fitted with Lankford coefficients with the ones fitted at small and large plastic strain levels.
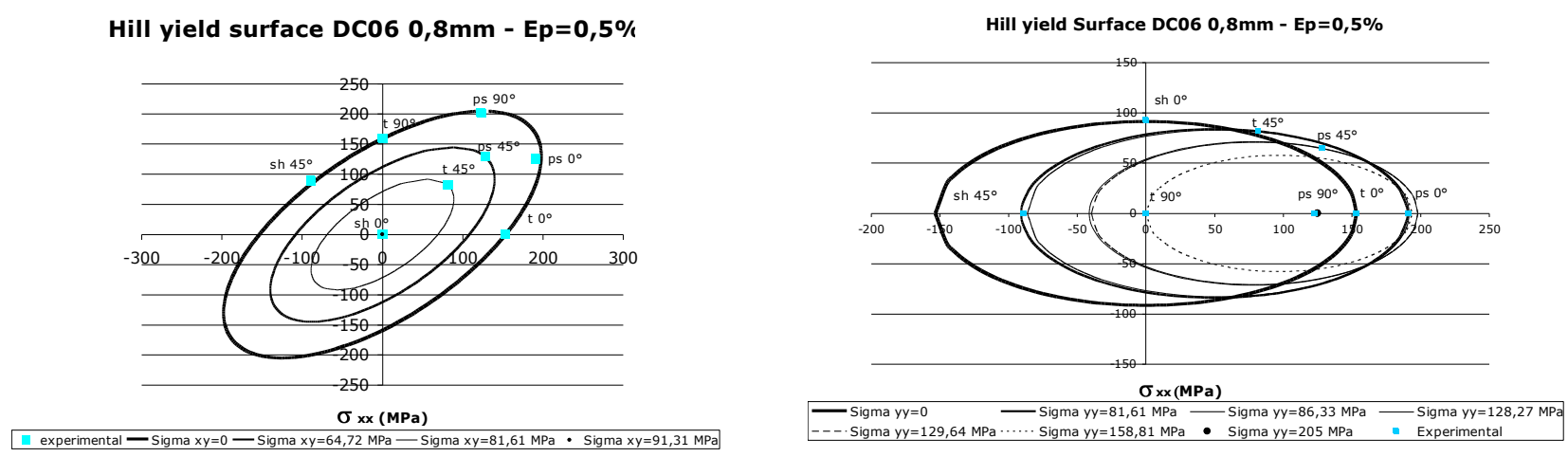

Figure 5: Hill yield surface fit with 8 mechanical tests.

Figure 7 shows the shape of the Hosford 1979 yield function fitted using Lankford coefficients and with 5 mechanical tests (tensile at RD and TD, plane-strain at RD and TD and simple shear at $45^{\circ}$ from RD) at two plastic strain levels.

Fig. 6 and Fig. 7 show the differences between the identification methods.

For Hill 1948 criterion (Fig. 6), the function fitted using a large plastic strain fit most of the experimental points at different plastic strain levels, while the one fitted at a small plastic strain should represent better the initial yield function. For both cases the flow rule of Eq. 3 is no longer respected for tensile stress-state, as it can be easily deduced from material parameters of Table 3 . Fitting material parameters using Lankford coefficients is not accurate for stress states others than tensile. 


\section{Hill 1948 DC06 0,8mm}

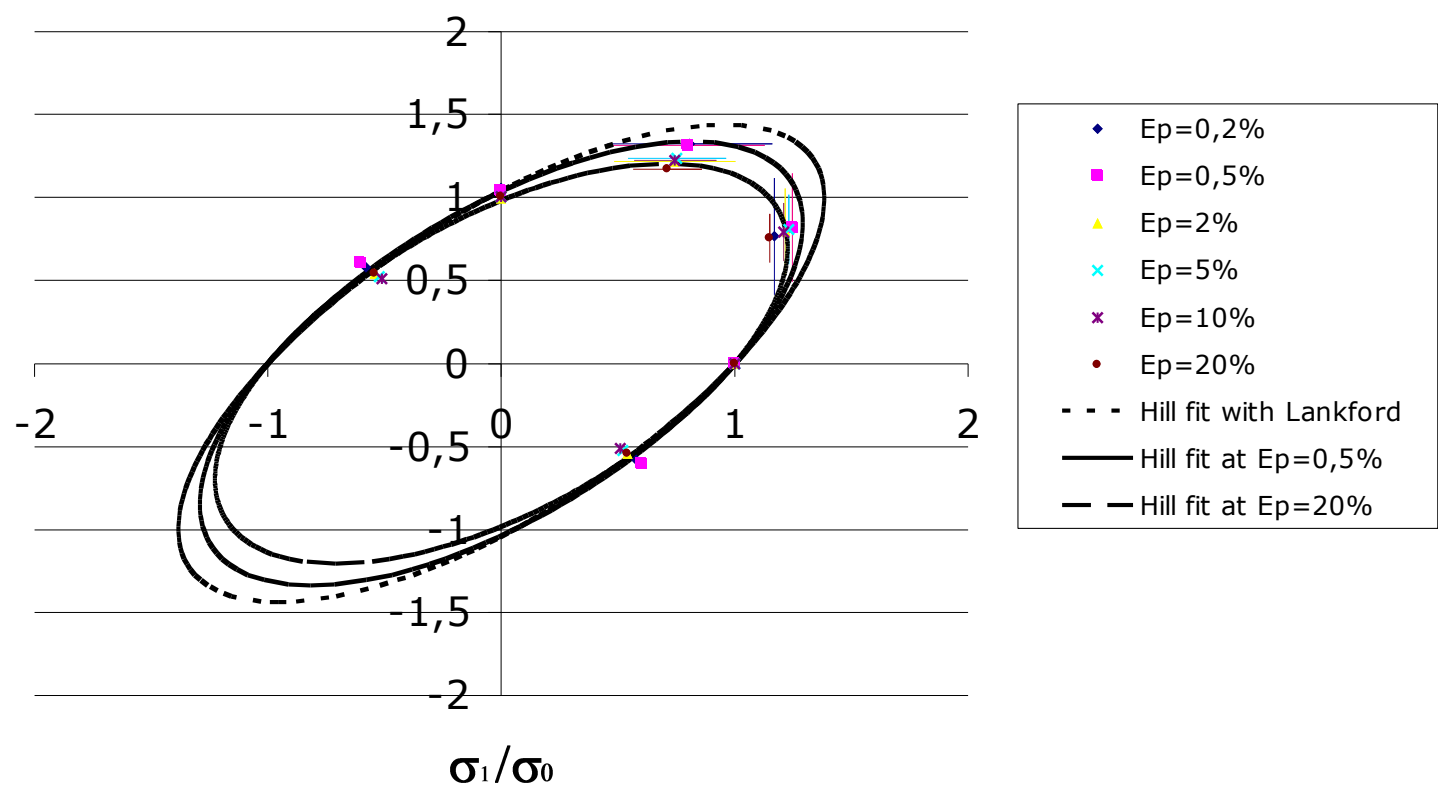

Figure 6: Shape of the Hill 1948 yield function.

\section{Hosford 1979 DC06 0,8mm}

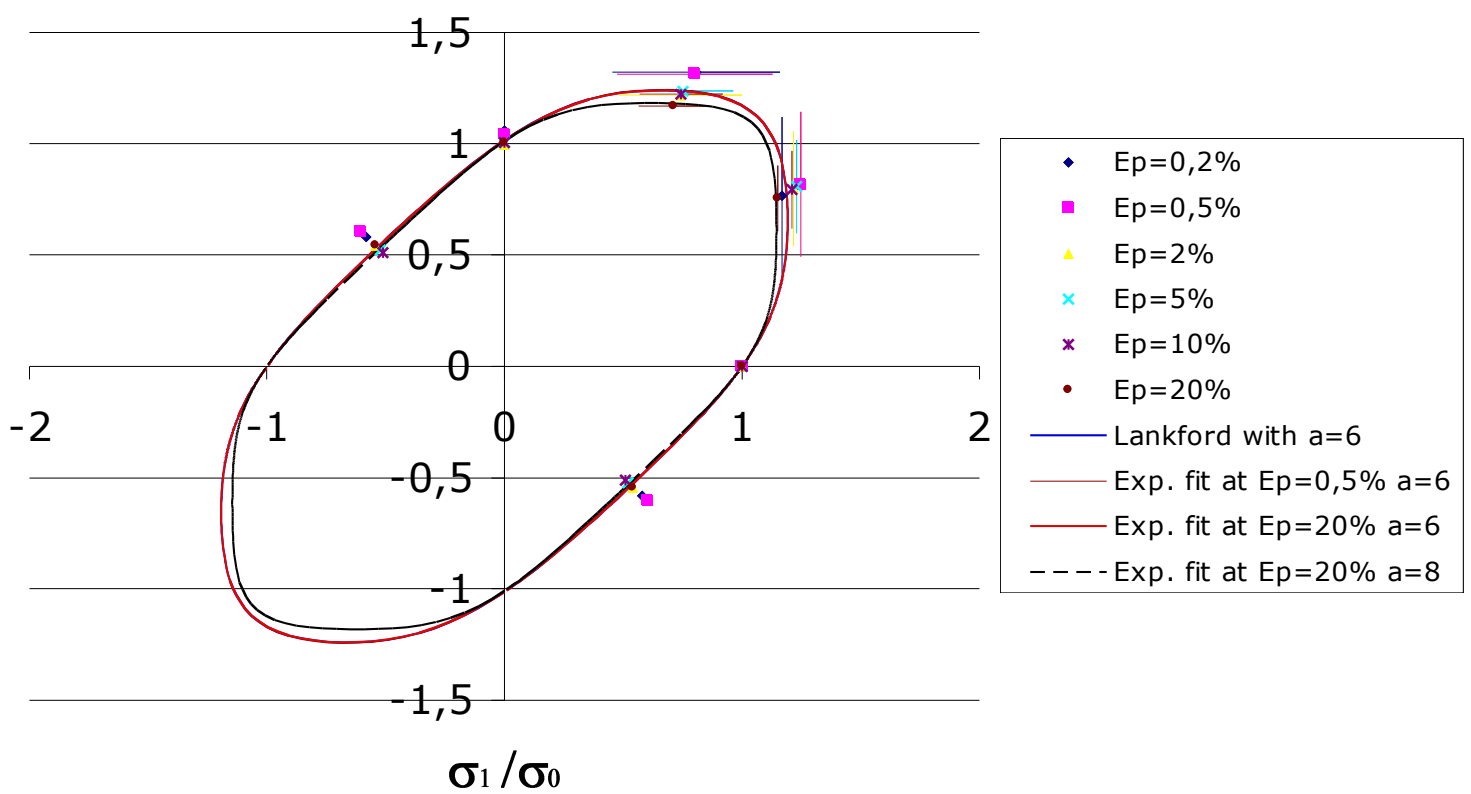

Figure 7: Shape of Hosford 1979 yield function.

Hosford 1979 criterion shows a high robustness thanks to parameter $a$. This parameter is imposed to be 6 (BCC material) when Lankford identification is used. When fitting experimental data for plastic strain levels from $0,2 \%$ to $10 \% a=6$ is obtained and the shape of the functions are the same as the one obtained using Lankford coefficients (Fig. 7). When fitting for a plastic strain of $20 \%$ the $a$ parameter is optimized to 8 and the shape differs from the other ones. In this last case the 
yield function shape is drawn again, keeping the materials parameters $H, F$ and $G$ but imposing the exponent $a=6$. It can be seen that this new shape coincides with the Lankford one. This apparent shape change it could be due to the influence of a back-stress (further study).

\begin{tabular}{|l|c|c|c|c|c|}
\hline Material parameters & $H$ & $F$ & $G$ & $N$ & $a$ \\
\hline Hill 1948 for $\mathrm{Ep}=0,5 \%$ & 1.22 & 0.64 & 0.78 & 2.82 & - \\
\hline Hosford 1979 for $\mathrm{Ep}=0,5 \%$ & 0.66 & 0.25 & 0.34 & - & 6 \\
\hline
\end{tabular}

Table 3: Material parameters for DC06 steel of $0.8 \mathrm{~mm}$ using yield stresses.

\section{Conclusions and perspectives}

This paper presents the identification of two yield criteria by two methods: one that takes into account strain measurements on tensile tests and the other one the stress measurement of three types of mechanical tests.

It is shown that Lankford coefficients are not enough to identify Hill 1948 yield criterion accurately and mechanical tests able to reproduce other stress states are required to obtain a better approach. It is also shown that fitting the exponent of the Hosford 1979 criterion ensures a good approximation to the experimental data.

It can also be seen that the plane-strain tests have a larger influence than the simple shear tests over the yield surface identification.

Further studies will focused on the identification of a proper yield function evolution law (hardening law).

\section{Acknowledgements}

As Research Associate of National Fund for Scientific Research (Belgium), A.-M. Habraken thanks this Belgian research fund for its support.

The authors also thank the Belgian Federal Science Policy Office (Contract P5/08) and Région Wallone for their support.

\section{References}

[1] P. Flores, E. Rondia and A.M. Habraken: Development of an experimental equipment for the identification of constitutive laws. Int. J. Form. Proc. To be published.

[2] H. Pijlman: Sheet material characterisation by multi-axial experiments Doctoral thesis (University of Twente, The Netherlands 2001).

[3] Z. Hu: Sheet Loi de comportement des metaux en grandes deformations tenant compte de l'évolution de la microstructure Doctoral thesis (Inst. Nat. Pol. de Grenoble, France 1992).

[4] B. Banabic, H.-J. Bunge, K. Pöhlandt and A.E. Tekkaya.: Formability of metallic materials (pp. 139-140. Springer 2000).

[5] W. Hosford: Materials Science \& Engineering A257 (1998) 1 - 8.

[6] T. Kuwabara, S. Ikeda and Y. Asano: Effect of anisotropic yield functions on the accuracy of springback simulation (pp. 887 - 892, NUMIFORM 2004). 